\title{
ÉDITORIAL
}

\section{«In manu imperiti est sicut gladius strictus in dextra furiosi »}

\author{
P. Goetz \\ (C) Lavoisier SAS 2015
}

Récemment je reçois à mon cabinet une patiente qui se plaint de l'ampleur prise par un eczéma sur son bras, et ce, malgré les soins qu'elle lui a prodigués. Elle a appliqué, comme elle l'a appris, une huile essentielle de pin à $1 \%$ diluée dans de l'huile d'amande douce. L'examen du bras montre qu'il y a un décollement de l'épithélium. Il s'agit en réalité d'une infection à staphylocoque. J'ai eu beau lui expliquer que l'application d'huile d'amande aussi douce soit-elle, sous un pansement occlusif, n'est pas un traitement d'une lésion ouverte du derme et qu'ajouter une huile essentielle n'arrange rien, ni l'allergie ni l'évitement d'une infection. Elle avait simplement appliqué ce qu'on lui avait enseigné.

Autre exemple : je suis attaché, quant à moi, au traitement de la dégénérescence cérébrale et j'introduis le plus souvent possible des « simples » pouvant être un traitement adjuvant important sur les symptômes de ce type de maladie.

Je reçois un patient qui me demande comment réajuster son traitement qui contient un anticholinestérasique. Depuis qu'il en prend, il a des tremblements, des crampes et une accélération de ses pensées. De plus il prend également depuis peu de la TM de petite pervenche et on lui a recom- mandé la TM d'Ignatia amara. Ignatia amara est le synonyme de Strychnos ignatii P.J. Bergius. La fève de Saint Ignace est toxique et, si elle fut employée à des doses de D3 au XIX ${ }^{\mathrm{e}}$ siècle, son utilisation en dehors de l'homéopathie doit être abandonnée aujourd'hui. Stein en 1797 qui prônait ses avantages dans des troubles gastriques et des spasmes de tout ordre, écrivait cependant « in manu imperiti est sicut gladius strictus in dextra furiosi » « Elle est, dans les mains d'un inexpérimenté, comme un glaive dans une main de fous $»$.

Il y a bien des nouveautés à faire connaître en premier aux spécialistes, mais il s'agit de prévenir des accidents après des enseignements mal compris ou des lectures d'utilisations anciennes.

Nous nous attachons dans notre Revue à ce que tout article publié ait été préalablement contrôlé par un membre du comité de rédaction ou du comité scientifique, afin qu'il n'y ait pas de mauvaises interprétations.

Rappelons encore que ce qui est établi in vitro ou sur l'animal est indicatif. Les expérimentations cliniques n'ont de force qu'après une étude confirmant la première étude.

P. Goetz $(\square)$

Médecin, enseignant de phytothérapie clinique,

faculté de médecine, Paris XIII, 93017 Bobigny cedex, France

e-mail : paul.goetz@wanadoo.fr 